\title{
Oculina patagonica, Scléractiniaire hermatypique introduit en Méditerranée
}

\author{
H. Zibrowius \\ Station marine d'Endoume; Marseille, France
}

\begin{abstract}
Oculina patagonica, a hermatypic scleractinian coral introduced to the Mediterranean Sea. A large colony of Oculina patagonica growing on a cliff (depth 0,5 to $2 \mathrm{~m}$ ) about $1 \mathrm{~km}$ from Savona harbour (Gulf of Genoa, Italy) was discovered in 1966 and was found prosperous and spreading when surveyed again in 1971 and 1972. Water temperature in the area was found to vary from about $11^{\circ} \mathrm{C}$ to about $26^{\circ} \mathrm{C}$; during the greater part of the year it remains far below temperatures generally considered necessary for growth and even survival of hermatypic scleractinians. In spite of the local pollution which favours dense and prosperous populations of barnacles and ascidians, the encrusting colony of $O$. patagonica (now measuring $1,2 \mathrm{~m} \times 1,5 \mathrm{~m}$ ) tends to cover these other organisms. The Savona record of $O$. patagonica is the first one of a living colony since nothing was known on the actual range of the species, probably living on the Atlantic coast of South America. Previously, O. patagonica was known only from worn fragments, fossil or subfossil, from Argentina. Liberation of larvae (one of which settled on the surveyed cliff) by a mature colony that came to Savona waters on a ship bottom, is the only acceptable explanation for the highly surprising arrival of the exotic scleractinian in the Northwestern Mediterranean. Transplantation of samples of $O$. patagonica broken off the Savona colony to clean water off Marseilles has proven very successful. The discovery of $O$. patagonica in the Mediterranean has provided material for a detailed redescription of the species and an opportunity for a systematic and biogeographic survey of the genus Oculina, as well as for remarks on hermatypic corals in extra tropical waters.
\end{abstract}

\section{INTRODUCTION}

Dans un article d'ordre zoogéographique sur la Méditerranée nord-occidentale, et en particulier sur la mer Ligure, Ross (1969, p. 151) remarquait qu'un Scléractiniaire du golfe de Guinée (São Tomé), Oculina arbuscula, aurait été introduit assez récemment dans le golfe de Gênes. Des colonies vivantes et propères de cette espèce auraient ainsi été trouvées à faible profondeur à Savona et à Portofino. On connaît bien des exemples d'espèces marines végétales et animales qui, transportées accidentellement par des bateaux, ont réussi à coloniser des aires géographiques nouvelles. Or, la remarque de Rossi sur l'introduction d'une espèce de Scléractiniaire exotique en Méditerranée semble être la première indication sur une introduction accidentelle d'une espèce de ce groupe dans une autre mer.

En réalité, l'introduction mentionnée par Rossı (1969) est encore plus remar- 
quable que ne le laisse deviner l'indication sommaire publiée à un endroit où elle aurait pu passer inaperçue. En effet, il y a eu introduction, dans le golfe de Gênes (à Savona, et non à Portofino) d'un Scléractiniaire hermatypique. Cependant, il s'agit de l'espèce Oculina patagonica ANGELIs, connue seulement d'après des échantillons fossiles et subfossiles de l'Argentine, et différente aussi bien de l'espèce américaine Oculina arbuscula AGAssiz, que d'une espèce ouest-africaine abusivement assimilée à cette dernière.

L'acclimatation de ce Scléractiniaire hermatypique en Méditerranée, espèce n'ayant jamais été récoltée vivante ailleurs, est si remarquable que l'histoire de sa découverte mérite d'être connue.

Le Scléractiniaire en question fut découvert en été 1966 par Lurgr Morra, membre du club de plongée de Turin (Circolo subaquei di Torino), lorsqu'il explorait les abords de la plage d'Albissola Marina, près de Savona. L. Morra en avait alors détaché quelques fragments afin de demander des renseignements à des naturalistes. Or, personne ne semblait connaître l'espèce qui l'avait fait penser à certains coraux exotiques figurés dans les revues de plongée. En général, il devait même rencontrer un certain scepticisme quant à l'authenticité de sa récolte. Ignorant que Rossx (1969, p. 151) avait fini par attribuer le corail de Savona à Oculina arbuscula, prétendue africaine, LUIG MoRrA cherchait toujours à obtenir des renseignements à son sujet. C'est ainsi que j'ai enfin pu entrer en contact avec lui, par l'intermédiaire de G. Scheer (Hessisches Landesmuseum, Darmstadt), auteur de divers articles sur des coraux récifaux actuels, et auquel L. MoRRA s'était également adressé.

Après avoir reçu à mon tour des fragments fraichement récoltés par L. Morra, des photos sous-marins et une description détaillée de la colonie ainsi que des peuplements qui l'entourent, nous avons décidé d'aller plonger ensemble sur les lieux. C'est ainsi que jai pu voir la colonie de l'énigmatique Scléractiniaire pour la première fois le 3 juillet 1971. J'y suis retourné le 23 octobre 1972, accompagné cette fois de J. G. Harmelin, de la Station marine d'Endoume.

\section{RESULTATS}

Le biotope à Oculina patagonica à Savona, caractéristiques hydrologiqueset peuplement benthique

Le groupe des écueils de la Madonnina est situé devant la côte rocheuse longée par la route entre le port de Savona et la plage de Albissola Marina, dans la partie ouest du golfe de Gênes. La direction générale de cette côte est sud-ouest / nord-est. Le groupe des écueils $\left(08^{\circ} 30^{\prime} \mathrm{E}, 44^{\circ} 19,5^{\prime} \mathrm{N}\right)$, bien plus proche de la plage de Albissola Marina $(0,3 \mathrm{~km})$ que du port de Savona $(1 \mathrm{~km})$ et s'étendant jusqu'à une centaine do mètres au large, comprend trois grands et plusieurs petits rochers de hauteur variée au-dessus de la surface de la mer. Le rocher le plus près de la côte est celui situé au sud $\mathrm{du}$ groupe; c'est d'ailleurs celui-ci, le plus élevé, qu'on aperçoit d'abord de la route venant de Savona. Le rocher le plus éloigné du rivage est celui du nord, vers la plage. Tous les rochers, bien visibles de la côte (affleurant à la sưrface ou émergés) 
ainsi que quelques rochers entièrement immergés, s'élèvent sur des fonds essentiellement rocheux et de galets d'environ 3 à $6 \mathrm{~m}$ de profondeur; plus au large, des fonds de sable vaseux succèdent aux fonds durs.

Les conditions hydrologiques précises dans ce secteur ne sont pas connues mais celles mesurées à la côte et à la surface à Varazze (à environ $10 \mathrm{~km}$ au nord-est de Savona) peuvent servir de repère, notamment en ce qui concerne la température de l'eau. Ainsi à Varazze, au cours d'une année, la température variait entre $11,1^{\circ} \mathrm{C}$ et $26^{\circ} \mathrm{C}$, la salinité entre $36,04 \%$ et $38,30 \%$, le pH entre 8,05 et 8,25 (13 mesures entre février 1970 et février 1971, données fournies par G. ReLINI).

Deux égouts municipaux débouchent au voisinage des écueils, l'un sur la côte même, l'autre - plus important et immergé - à une certaine distance du rivage. Il semble que le premier fonctionne depuis 1945 environ, l'autre depuis environ 1960. Ces égouts amènent dans la mer les eaux domestiques d'un quartier d'habitation voisin de la ville de Savona. Si la pollution, au voisinage immédiat des écueils, n'est donc pas d'origine industrielle directe, le déversement de détergents etc. par l'intermédiaire des eaux ménagères n'est probablement pas négligeable à côté des constituants organiques de cette pollution (excréments, etc.). On doit noter également que l'entrée nord de l'important port de Savona est distante d'environ $1 \mathrm{~km}$. Même par mer très calme l'eau au voisinage des écueils est constamment trouble car chargée de particules de détritus apportés par les égouts très proches. Temporairement exposés à une houle du large, les faibles fonds durs séparant les écueils ne peuvent pas s'envaser de façon durable, mais, au-delà des faibles fonds rocheux, les apports des égouts ont provoqué un envasement des fonds sableux et la disparition des herbiers de Posidonies dont on ne trouve que des rhizomes morts.

Etant donné cette pollution locale, il va de soi que le peuplement benthique des écueils est sensiblement modifié par rapport au peuplement naturel à algues photophiles qui, en eau pure, convrirait des substrats rocheux analogues. A en juger par l'analyse des échantillons prélevés, cette modification est plus évidente au niveau des espèces déterminant l'aspect macroscopique du peuplement qu'au niveau de la faune vagile qui lui est inféodée. Cette dernière est encore bien variée et comprend, notamment parmi les Polychètes et les Amphipodes, d'assez nombreuses espèces «banales» infralittorales, espèces en général liées aux algues photophiles et aux herbiers de Posidonies.

Les espèces animales relevées sur les écueils sont rassemblées çi-dessous.

Spongiaires: Leuconia sp., Spongia virgultosa ScHMIdT.

Hydraires: Aglaophenia pluma (LINNÉ), Eudendrium capillare ALDER, Halecium mediterraneum WeISMANn, Obelia dichotoma (LINNÉ).

Alcyonaires: Cornularia cornucopiae PaLLAs.

Actiniaires: espèce non identifiée.

Mollusques Placophores: Chiton olivaceus SPENGLER.

Mollusques Gastéropodes: Ammonicera fischeriana (Monterosato), Alvania cimex (LINNÉ), Bittium reticulatum DA CosTa, Columbella rustica (LinNé), Diodora gibberula (LAmarck), Murex trunculus Linné, Nassarius incrassatus (O. F. Müller), Patella caerulea Linné, Pisania orbignyi Pavraudeau, Putilla semistriata (MonTagu), Raphitoma leupbroyi (Mrchaud), Rissoa lineolata (Mrchaud), Trifora perversa 
(Linné), Tritonalia edwardsii (PAyRaudeau), Tritonalia erinacea (LinnÉ), Turboella parva (DA CosTA), Vermetus cristatus Brond, Vermetus triqueter Brvone.

Mollusques Pélécypodes: Anomia ephippium LinNé, Arca noae Linné, Arca lactea Linné, Brachidontes minimus (PoLr), Cardita calyculata Linnte, Chlamys multistriata (Pol), Hiatella arctica (LINNÉ), Musculus costulatus (RIsso), Musculus marmoratus (Forbes), Mytilus galloprovincialis LAMARck, Ostrea edulis LinNÉ.

Polychètes autres que Serpulidae: Amphiglena mediterranea (LEYDrG), Amphitrite rubra (Risso), Audouinia filigera (Delle ChIAJE), Cirratus cirratus (O. F. MüLLER), Eunice harassii Audouin \& MrlNe EdWARds, Eunice siciliensis Grube, Harmothoe areolata GRU'Be, Harmotboe spinifera (EHLERs), Lepidonotus clava (MoNTAGU), Lumbrineris coccinea (RENIER), Lumbrineris funchalensis (KINBERG), Lysidice ninetta Audoun \& Mrune Edwards, Nereis costae Grube, Nicolea venustula (Montagu), Pbyllodoce paretti (BlaINville) ou Phyllodoce rubiginosa SAINT-Joseph, Platynereis dumerili (Audourn \& Milne Edwards), Polycirrus sp., Polydora cf. ciliata (Johnston), Polyophthalmus pictus (Dujardin), Sabellaria spinulosa (LeUckarT), Thelepus cincinnatus (FABRICIUs), Trypanosyllis zebra Grube.

Polychètes Serpulidae: Filograna sp., Hydroides diantbus (Verrill), Hydroides elegans (HAswelL), Hydroides pseudouncinata Zibrowius, Pileolaria militaris ClaParède, Pomatoceros lamarckii (Quatrefages), Pomatoceros triqueter (Linné), Protula sp., Serpula concharum Langerhans, Spirobranchus polytrema (PhILIPPI).

Cirripèdes: Balanus perforatus BrugIÈrE, Balanus trigonus Darwin.

Décapodes: Alpheus dentipes Guerin, Athanas nitescens (LEACH), Clibanarizs erytbropus (LATReIlle), Galatbea dispersa (BATE), Maia verrucosa (MILne EDwards), Pagurus anachoretus Risso, Pilumnus birtellus (Linné), Pisidia bluteli (Risso), Pisidia longimana (Risso), Thoralus cranchi (LEACH).

Tanaidacés: Antanais sp., Leptochelia savignyi KroYer.

Isopodes: Anthuridae et Janiridae non identifiés, Paranthura sp.

Amphipodes: Coropbium acherusicum Costa, Dexamine spinosa (Montagu), Elasmopus rapax A. Costa, Eusiroides dellavallei Chevreux, Jassa falcata (MonTAGU), Leucotboe spinicarpa (ABILdgaArd), Maera inaequipes (A. Costa), Orchomene humilis (A. Costa), Perioculodes longimanus (BAte \& Westwood), Podocerus variegatus LEACH, Stenotboe monoculoides (MONTAGU), Stenotboe spinimana Chevreux.

Pycnogonides: Anoplodactylus pygmaeus Hodge, Tanystylum orbiculare WrLson. Sipunculides: Phascolosoma granulatum LEUCKART.

Bryozoaires: Scbizobrachiella sanguinea (Norman), Scbizoporella longirostris Hincks, Schizoporella errata (WATERs), Turbicellopora magnicostata (BARRoso), Watersipora subovoidea (ORBIGNY).

Echinodermes: Arbacia lixula (Linné), Asterina gibbosa (Pennant), Opbiotrix fragilis (AbIldgaArd), Paracentrotus lividus Lamarck.

Ascidies: Microcosmus exasperatus (Heller).

La flore algale des rochers mériterait d'être étudiée en détail. L'examen préliminaire de quelques échantillons prélevés au hasard a déjà donné des résultats intéressants. Une grande algue verte, Codium decorticatum, est une espèce plutôt rare en Médițerranée et inçonnue dans le golfe $\mathrm{du}$ Lion, mais très répandue danş les mers 
chaudes. Une algue verte filamenteuse du genre Derbesia est une espèce non signalée en Méditerranée. L'algue brune, Dictyota cf. dicbotoma se distingue de la forme habituelle; il pourrait s'agir, tout au moins, d'une morphose particulière due à ce milieu pollué. Enfin, un petit gazon rouge assez abondant est constitué par un mélange de diverses espèces: Antithamnion elegans, Bryopsis sp., Cladopbora sp., Erytbrotrichia carnea, Gelidium spatulatum, Lomentaria sp., Sphacelariasp.

L'aspect général du peuplement benthique des écueils est déterminé par l'abondance de quelques espèces animales et végétales: Mytilus galloprovincialis (dans le niveau le plus élevé), Balanus perforatus, Microcosmus exasperatus, Schizoporella errata, Lithophyllum incrustans, Dictyota cf. dichotoma.

Dans les niveaux inférieurs des rochers, à quelques mètres de profondeur, le peuplement est absolument dominé par Balanus perforatus et Microcosmus exasperatus. Toutefois, ces deux espèces sont déjà présentes, mais moins prépondérantes, dans le niveau superficiel à Mytilus galloprovincialis. M. exasperatus atteint une abondance extrême au point de constituer de véritables grappes de spécimens agglomérés. Selon C. Monniot' (in litt.) il s'agit d'une espèce très répandue dans toutes les mers tropicales, en particulier dans les eaux portuaires et sur les coques des bateaux. La présence de cette espèce en Méditerranée ne semble pas avoir été signalée auparavant. Toutefois, Monnor l'avait déjà reconnue dans une collection d'Ascidies du port de Bizerte (Tunisie).

En très faible proportion, une deuxième espèce de Cirripède, Balanus trigonus, est mélangée à Balanus perforatus, espèce dominante. B. trigonus est assez peu connu en Méditerranée, mais peut y avoir une certaine importance dans les peuplements portuaires (RELINI 1968). Dans les archipels de Madère et des Açores (campagnes du "Jean Charcot» en 1966 et 1971 ) cette espèce est très répandue dans les peuplements infralittoraux en eau pure.

Parmi les Serpulidae, Hydroides elegans et Hydroides dianthus ont été reconnues comme étant des espèces originaires d'autres mers et introduites en Méditerranée sur des coques de bateaux (Zibrowrus 1973).

C'est dans le groupe des écueils de la Madonnina, dans ce milieu assez particulier, que prospère la colonie de Oculina patagonica découverte par L. Morra en 1966. Elle y occupe une partie de la face nord d'un petit rocher entièrement immergé à proximité du deuxième grand rocher (vu de la plage d'Albissola Marina). Le sommet de ce petit rocher se trouve à un demi mètre environ sous la surface de la mer (profondeur variable suivant la pression atmosphérique, les marées, le vent, etc.), sa base vers $3 \mathrm{~m}$.

Entourée par le peuplement caractérisé ci-dessus, la colonie de O.patagonica occupe une zone continue, vaguement pentagonale qui mesure environ $1,2 \mathrm{~m}$ de haut et $1,5 \mathrm{~m}$ de large. Subhorizontale en haut, cette zone s'incline de plus en plus vers le bas jusqu'à atteindre la verticale dans sa partie inférieure. De chaque côté, un coin de la colonie déborde d'une arrête de la roche assez bien marquée. En haut, la colonie n'a pas atteint le sommet du rocher qui est couvert d'une moulière à Mytilus galloprovincialis. La limite inférieure, subhorizontale, de la colonie se situe vers $2 \mathrm{~m}$ de profondeur. En bas et sur les côtés, la colonie se trouve au contact d'un peuplement à dominances de Balanus perforatus et de Microcosmus exasperatus. Le Scléractiniaire est plus compétitif que les Ascidies et les Balanes, pourtant très prospères, puisque lẹ 
bord périphérique mince de la colonie s'étend sur ces autres organismes qu'il tend à recouvrir. Les Ascidies au contact du Scléractiniaire semblent être plus rapidement éliminées que les Balanes. Ces derniers peuvent rester vivants dans la zone périphérique de la colonie, tout en étant entièrement recouverts par celle-ci. On peut ainsi observer des cirres de Balanes en action sortant des petits orifices laissées par le Scléractiniaire. Plus vers l'intérieur de la colonie, c'est à dire, dans ses zones plus anciennes et le plus souvent considérablement épaissies, les Balanes sont en général morts. Certaines excroissances irrégulières et particulièrement marquées de la colonie, pour le reste plutôt encroûtante, correspondent d'ailleurs à des agglomérations de Balanes étouffées et recouvertes. Dans certains secteurs, la colonie comporte toutefois de véritables petites branches, irrégulières, courtes et assez massives, construites entièrement par le Scléractiniaire. Ainsi, dans l'ensemble, l'aspect de la colonie varie considérablement d'un secteur à l'autre, sans qu'une corrélation entre l'exposition et la forme soit évidente: il y a des zones plates, uniformes, des zones marquées par des bosses arrondies, et des zones à branches plus distinctes.

Le problème de l'arrivée du Scléractiniaire à Savona

Il est exclu que $O$. patagonica soit un élément autochthone de la faune méditerranéenne et que cette espèce coloniale, vivant obligatoirement à faible profondeur à des endroits bien éclairés (présence de zooxanthelles), ait pu échapper aussi longtemps à l'attention des plongeurs, biologistes ou non, si nombreux à explorer la Méditerranée. On doit exclure également l'éventualité de la survivance, uniquement à Savona dans un endroit aussi particulier, d'une espèce qui aurait été plus répandue dans le bassin méditerranéen à des époques géologiques plus ou moins anciennes. On ne connaît, par ailleurs, dans les dépôts tertiaires ou quaternaires du pourtour méditerranéen, aucune forme fossile que l'on pourrait rapprocher du Scléractiniaire de Savona. Ainsi sa présence à Savona peut s'expliquer uniquement par une introduction assez récente.

L'unique colonie du Scléractiniaire vit à proximité $(1 \mathrm{~km})$ de l'important port de Savona et diverses autres espèces non originaires de la Méditerranée susceptibles d'être transportées sur des coques de bateaux y ont été reconnues (voir plus haut). Toutefois, une coincidence extraordinaire de facteurs doit avoir été à l'origine de l'installation et de la réussite de cette colonie. Le fait que la colonie ait continué de prospérer et de s'étendre dans ce milieu de plus en plus pollué en l'emportant sur les Balanes et les Ascidies, est aussi remarquable que son installation.

Les Scléractiniaires ont, en général, la réputation d'être très sensibles à d'importantes variations de la salinité et à tous les aspects de la pollution auxquels ils s'exposeraient presque sûrement en s'installant sur les coques de bateaux: les ports fréquentés sont toujours plus ou moins pollués, et très souvent caractérisés par des eaux à salinité variable ou constamment inférieure à celle de l'eau de mer normale. C'est ainsi que les Scléractiniaires sont inconnus dans les peuplements habituels de salissures couvrant les coques de bateaux en service. Par contre, on s'imagine plus facilement qu'un Scléractiniaire puisse coloniser la coque d'un bateau hors service, 
mouillé pendant longtemps en rade, avant que l'épave ne soit acheminée vers une usine de démolition d'un centre sidérurgique (comme il en existe dans le golfe de Gênes, et en particulier à Savona).

La vie larvaire libre des Scléractiniaires semble en général d'une courte durée, et l'émission de larves viables n'a pas lieu à une salinité et à une température quelconques de l'eau; on connaît toutefois le cas de larves pouvant flotter, passivement, pendant deux à trois semaines (ABE 1937, 1939). Quoiqu'il en soit, le fait improbable s'est produit: une colonie de O. patagonica en maturité sexuelle a dû arriver dans les parages de Savona et une de ses larves s'installer sur le rocher.

Quant à l'origine de la colonie mère, arrivée dans les parages de Savona pour libérer la larve qui devait donner la présente colonie, on se trouve réduit aux hypothèses les plus vagues. O. patagonica, connue à l'état fossile et subfossile en Argentine, pourrait vivre sur les côtes atlantiques de l'Amérique du Sud, côtes qui sont encore largement inexplorées. Ou l'espèce existerait-elle également sur les côtes atlantiques de l'Afrique? Certaines espèces de Scléractiniaires hermatypiques, aussi bien que d'autres invertébrés sessiles, sont en effet communes à la faune des Antilles et à celle de l'Atlantique africain tropical, en particulier à cellé des îles du Cap Vert (LABorel 1973). Notons à titre de curiosité que LE CoInTre $(1950,1952)$ avait même reconnu des Gastéropodes «chilopéruviens» dans le Quaternaire ancien (Calabrien) du Maroc.

\section{Transplantation expérimentale d'échantillons de Savona à Marseille}

Des échantillons prélevés sur la colonie de O. patagonica à Savona le 23 octobre 1972 ont été ramenés vivants à Marseille dans un grand bidon rempli d'eau de mer puisée à Savona face aux écueils; pendant le voyage en voiture d'environ huit heures le bidon était dépourvu de tout dispositif d'aération et d'isolation thermique. Mis dans l'aquarium de la Station marine d'Endoume (alimenté en eau puisée devant le laboratoire dans l'anse des Cuivres) tard le soir, tous les fragments avaient leurs polypes parfaitement épanouis le lendemain matin.

Les plus petits fragments apportés ont été laissés dans cet aquarium tandis que les plus grands fragments, mesurant environ 5 à $20 \mathrm{~cm}$, étaient destinés à être installés en eau pure et en eau polluée dans les parages de Marseille. Au moyen de fils en nylon, ces échantillons furent solidement attachés à des plaques en plexiglass de récupération (perforées à divers endroits pour faire passer les fils croisés au-dessus des échantillons). A cette manipulation grossière, les échantillons, plus ou moins émergés, ont réagi par une forte sécrétion de mucus; ils s'en sont remis après quelques heures dans l'aquarium (polypes parfaitement épanouis).

Le 25 et le 26 octobre, les échantillons ainsi attachés à un support furent installés en milieu marin: les uns devant la Station marine d'Endoume, dans l'anse des Cuivres, à proximité de la prise d'eau de l'aquarium, les autres dans l'eau pure du large à la face sud de l'île de Riou (rocher "Impériou de terre»; $43^{\circ} 10,3^{\prime} \mathrm{N}, 05^{\circ} 23,6^{\prime} \mathrm{E}$ ).

Le jour prévu pour l'installation des échantillons à l'île de Riou, la mer était agitée et il était impossible de fixer, comme prévu, les plaques, dans un endroit bien 
éclairé, à faible profondeur. Les plaques ont dû être abandonnées, au contraire, à des endroits relativement abrités et moins éclairés, vers 15 à $17 \mathrm{~m}$ de profondeur, dans des peuplements à Gorgones [Eunicella cavolini ( $\mathrm{KocH}$ ) et Paramuricea clavata (Risso)] et rares algues sciaphiles, à la face nord du rocher. Les échantillons ainsi installés ont été contrôlés à des intervalles irréguliers, la dernière fois en janvier 1974. Ils étaient alors toujours en bon état, les polypes parfaitement épanouis. Les fils en nylon, croisés en divers sens sur les échantillons, ont été progressivement recouverts par les tissus vivants et le squelette, au point de ne plus être visibles. Les échantillons ont commencé à se souder aux plaques servant de substrat, et le plus grand d'entre eux, particulièrement prospère, a même sensiblement allongé quelques petites excroissances. Malgré l'éclairement réduit à cette nouvelle station (profondeur, face nord, zones subverticales à surplombantes) par rapport à celui de l'emplacement originel à Savona, la couleur des tissus vivants n'a pas sensiblement changé; l'éclairement reçu semble donc encore suffisant pour les zooxanthelles.

Dans l'anse des Cuivres les plaques portant les échantillons furent implantés entre 2 et $5 \mathrm{~m}$ de profondeur. Cette nouvelle station ressemble davantage à celle de la colonie de Savona, par l'éclairement reçu (faible profondeur) aussi bien que par les peuplements dérivés des peuplements à algues photophiles, à la suite de la pollution locale (petits égouts débouchant à proximité). Les eaux très polluées de l'Huveaune, petite rivière débouchant à $2,5 \mathrm{~km}$ de distance à la plage du Prado et drainant les eaux d'une zone industrielle, transgressent fréquemment jusque devant l'anse des Cuivres. L'ensemble du golfe de Marseille est d'ailleurs sensiblement pollué (ports, égouts), en particulier la zone côtière devant la ville.

Pendant plusieurs semaines, les échantillons installés dans l'anse des Cuivres et ceux, de plus petite taille, gardés dans l'aquarium de la Station marine d'Endoume (alimenté en eau de l'anse des Cuivres) se portaient bien, à en juger par les polypes épanouis. Cependant, l'état de tous ces échantillons changeait bursquement à la suite de quelques tempêtes d'hiver (vent et houle du secteur sud-est à sud-ouest). Les polypes furent alors rarement observés en extension, les tissus vivants entre les calices étaient en régression, et certaines zones se trouvaient complètement dénudées de tout tissu vivant pour être rapidement envahies, surtout sur les échantillons dehors, par des algues vertes filamenteuses. Si elle n'était pas rapidement bouchée ou désamorcée, la pompe envoyait, lors des tempêtes, une eau très chargée de fines particules dans l'aquarium. Le déclin des échantillons, aussi bien dans l'aquarium que dehors, semblait ainsi due, essentiellement, à la fraction fine du sédiment soulevé; tout effet abrasif de la fraction grossière qui aurait pu intervenir dans l'anse des Cuivres, était évidemment exclu dans l'aquarium. La forte absorption connue de détergents et d'autres substances était peut-être la cause de la nocivité de la fraction argileuse pour les échantillons.

A partir du mois de janvier 1973, les petits échantillons de O. patagonica, en plein déclin dans l'aquarium de la Station marine d'Endoume, ont été transférés, successivement, dans un aquarium fonctionnant en circuit fermé avec de l'eau pure du large (mis au point par PoIzAT 1972). En quelques jours, les échantillons avaient visiblement récupéré et les polypes étaient à nouveau en parfaite extension tandis que les tissus vivants commençaient à recouvrir le coenosteum dénudé entre les calices.

Afin d'arrêter le déclin des échantillons installés dans l'anse des Cuivres, il 
paraissait souhaitable de les transférer dans l'eau pure du large (île Riou) où d'autres échantillons, également installés depuis le début de l'expérience, étaient restés prospères. Le transfert des échantillons de l'anse des Cuivres (à l'exception d'un échantillon témoin laissé sur place) à l'île Riou a eu lieu le 12 mars 1973. Les échantillons avec leurs plaques furent installés, les uns dans des peuplements sciaphiles entre 9 et $15 \mathrm{~m}$ de profondeur, à proximité des échantillons déjà en place, les autres à $10 \mathrm{~m}$ de profondeur en pleine lumière, dans un peuplement à algues photophiles (Codium et Asparagopsis). Visités un mois après ce transfert (le 13 avril 1973), les échantillons étaient en bien meilleur état que précédemment dans l'anse des Cuivres, leurs polypes à nouveau épanouis. Au cours des mois suivants, les fils en nylon tenant les échantillons sur les plaques servant de substrat, furent progressivement recouverts par les tissus vivants et le squelette, et les échantillons commençaient à se souder aux plaques. Après un certain temps, ces échantillons étaient ainsi à nouveau aussi prospères et en pleine croissance que ceux installés à l'île de Riou dès le début de l'expérience.

Après leur déclin au début de l'année 1973, un seul des échantillons dans l'anse des Cuivres a été laissé sur place comme témoin. Au cours de d'été, il commençait à récupérer, semblable à ceux transférés dans l'eau pure du large, et en septembre 1973 il était soudé à la plaque servant de substrat. Il a continué à s'étaler latéralement, la partie encroûtante atteignant par endroit $10 \mathrm{~mm}$ de large en janvier 1974. Pendant ce deuxième hiver que l'échantillon est implanté dans l'anse des Cuivres, le déclin spectaculaire de l'hiver précédent ne semble pas se reproduire.

Poursuivie pendant déjà 15 mois, cette expérience de transplantation de O. patagonica montre que l'espèce peut bien vivre dans la région de Marseille malgré des températures hivernales parfois encore inférieures à celles notées dans la région de Savona. A Marseille, l'espèce semble trouver de bonnes conditions dans l'eau pure $\mathrm{du}$ large, mais temporairement précaires dans la zone polluée du golfe.

A titre de comparaison il est intéressant de noter que les Scléractiniaires méditerranéens autochthones avaient déjà disparu de l'anse des Cuivres et de ses abords, telles que les espèces photophiles Balanophyllia verrucaria (PALIAs) et Balanophyllia regia Gosse, qui y ont encore existé il y a moins de 20 ans. De même, aucune autre espèce de Scléractiniaire méditerranéenne testé n'a aussi bien supporté les conditions précaires dans l'aquarium de la Station marine d'Endoume.

\section{Scléractiniaires hermatypiques dans des eaux extratropicales}

On a pensé très longtemps que les Scléractiniaires hermatypiques vivaient exclusivement dans les eaux chaudes des mers tropicales. C'est seulement à une époque récente qu'on s'est aperçu de l'inexactitude de cette idée. Wells (1957, p. 1088) admettait déjà que les coraux récifaux pourraient survivre, pendant un certain temps, à des températures aussi basses que 16 à $17^{\circ} \mathrm{C}$. Macintyre \& Pilkey (1969) et Macintyre (1970) ont décrit le cas de deux espèces hermatypiques, prospères, sous forme de grandes colonies isolées, dans des fonds de 20 à $40 \mathrm{~m}$ devant Onslow Bay (North Carolina), dans des eaux où la température hivernale est inférieure à $16^{\circ} \mathrm{C}$ 
pendant trois mois et où elle peut accuser des minima de $10,6^{\circ} \mathrm{C}$. Les espèces en question sont Solenastrea hyades (DANA) et Siderastrea siderea (Ellis \& Solander), connues pour leur abondance dans les récifs des Caraibes, des Antilles et de Floride. Ce cas particulier n'est pas aussi isolé que cela pourrait paraître car les auteurs ont pu se référer à des publications faisant état d'observations analogues au sud de l'Australie. Le cas de Oculina patagonica, Scléractiniaire hermatypique ayant réussi à s'implanter à Savona $\left(44^{\circ} 19,5^{\prime} \mathrm{N}, 08^{\circ} 30^{\prime} \mathrm{E}\right)$, ne correspond pas à un phénomène observé pour la première fois.

Dans la région de Savona, treize mesures effectuées au cours d'une année indiquent une variation de la température de l'eau de mer entre environ $11,1^{\circ} \mathrm{C}$ et $26^{\circ} \mathrm{C}$ (voir plus haut). L'écart entre le minimum et le maximum annuels absolus est sans doute encore légèrement plus grand. Pendant la plus grande partie de l'année les températures sont donc de loin inférieures à celles autrefois considérées comme nécessaires pour la prospérité et mêtme la survie de Scléractiniaires hermatypiques. Or, les observations montrent que ces températures sont bien compatibles avec la prospérité et la croissance de $O$. patagonica.

Il ne faut d'ailleurs pas perdre de vue que la faune méditerranéenne actuelle posséde bien un Scléractiniaire hermatypique colonial, Cladocora caespitosa LiNNé, espèce dont les zooxanthelles viennent d'être étudiées par Duclaux \& Lafargue (1973). C'est une espèce typiquement infralittorale répandue dans tous les secteurs de la Méditerranée. Je l'ai trouvée particulièrement abondante et prospère sur les côtes de Tunisie, aux eaux relativement chaudes, de mêtme que dans le nord de l'Adriatique où la température hivernale de l'eau peut rester en dessous de $10^{\circ} \mathrm{C}$ pendant des semaines sinon des mois. De même, LaBorel (1961) a rapporté le cas d'un concrétionnement étendu à Cladocora caespitosa de la mer Egée. Sur les côtes de Tunisie, des colonies de $C$, caespitosa de plus d'un mètre de diamètre et de plusieurs dizaines de centimètres d'épaisseur au centre, ne sont pas exceptionnelles. Il semble toutefois que C. caespitosa ait eu des époques de prospérité encore plus grande en Méditerranée où elle formait alors, sinon de vrais récifs, tout au moins des bancs étendus. Ainsi Poizat (1970, p. 270) a fait état des «oncoides» à Cladocora, véritables dômes étendus, dans le golfe de Gabès. Sur la côte est du cap Bon (Tunisie), au nord de Nabeul, j'ai vu d'importants gisements de Cladocora datant peut-être du Tyrrhénien.

\author{
Le genre Oculina- \\ aperçu systématique et biogéographique
}

La systématique du genre Oculina est très confuse. Le genre est l'un de ceux établis par Lamarck (1816) et redéfinis par Mitne Edwards \& Hatme qui n'y ont gardé que deux des neuf espèces de Lamarck: O. virginea (considérée comme identique à Madrepora virginea Linné 1758) et O. diffusa Lamarck 1816. Minne EDwards \& HaIme (1850b, p. 6) ont choisi O. virginea sensu LAMARCK comme espèce type et l'ont redécrite d'après divers échantillons, dont celui de Lamarck (Milne EDwards \& Hame 1850b, 1857). Les échantillons de la collection Lamarck (O. virginea, O. dif- 
fusa) ont été mentionnés par RANSoN (1943) et devraient encore exister au Muséum National d'Histoire Naturelle, à Paris. Selon Vaughan \& Wells (1943) et Wells (1956), O. virginea sensu LAMARck serait la même espèce que O. diffusa LAMARck, mais différerait de Madrepora virginea LINNÉ 1758.

Mulne Edwards \& HaIme ont présenté $O$. virginea comme une espèce de l'océan Indien tandis que Lamarck avait indiqué «habite l'océan des Deux-Indes, la Méditerranée». Or, dans les temps anciens, il y a eu beaucoup de confusions au sujet de la provenance des objets d'histoire naturelle, souvent très recherchés, et également au sujet des «Deux-Indes» («orientales» et «occidentales»). Tout laisse à penser que les échantillons de O. virginea étudiés par LAMARCK et par Milne Edwards \& Harme provenaient des Antilles, tout comme ceux de l'espèce $O$. diffusa, reconnue, elle, comme américaine. La localité type de l'espèce Oculina fissipara MrLne Edwards \& HAIme 1850b, avait été indiquée comme étant le «cap Natal», localité cherchée ultérieurement tantôt en Afrique, tantôt au Brésil (voir plus loin). Les quatres autres espèces actuelles, $O$. banksi, $O$. petiveri, $O$. speciosa, $O$. valenciennesi, toutes décrites dans la même publication, étaient de provenance inconnue, mais divers auteurs ont par la suite signalé certaines d'entre elles aux Antilles.

Selon Milne Edwards \& Haime, O. varicosa Lesueur 1820, et O. pallens EhrenBERG 1834, toutes les deux des Antilles, seraient synonymes de O. diffusa LAMARCK, mais certains auteurs plus récents n'ont pas accepté cette mise en synonymie. Diverses autres espèces furent décrites en provenance de l'Atlantique américain (Antilles, Floride, Bermudes, South Carolina) dont certaines par la suite mises en synonymie entre elles ou avec des espèces plus anciennes: O. bermudiana Duchassaing \& Michelotti 1864, O. disticha Pourtalès 1869, O. tenella Pourtalès 1871, O. robusta Pourtalès 1871, O. cubaensis Duncan 1876, O. arbuscula Agassiz 1880, O. implicata Agassiz 1880, O. coronalis Quelch 1886, O. recta Quelch 1886.

VAUGHAN (1901, p. 194) et VERRILL (1901, p. 172) ont déjà reconnu la nécessité de reviser les nombreuses espèces américaines du grenre Oculina dont les descriptions, souvent insuffisantes, attribuaient en général trop d'importance à la forme de la colonie. THIEL (1941) a tenté cette révision en référant la plupart des formes décrites en provenance des mers américaines (ou qui y avaient été signalée par la suite) à seulement deux espèces: $O$. virginea et $O$. diffusa. Or, le fait que THIEL n'ait pas reconnu l'espèce Oculina tenella mais l'ait confondue avec Lophobelia tenuis Moseley (en réalité référable au genre Madrepora) n'est peut-être pas la meilleure référence.

Pour la première fois, l'existence du genre Oculina en dehors des mers américaines fut indiquée par Gravier (1909) qui rapportait une espèce du golfe de Guinée (São Tomé) à l'espèce américaine O. arbuscula AGassiz (localité type: South Carolina). Gravier fut suivi, pour cette identification, par Threl (1928) qui signalait la même forme à Fernando Po. Monod (1954), en étudiant du matériel du Caméroun, contestait cette identification et rapportait $O$. arbuscula sensu GRAvIER et THIEL à O. fissipara Milne Edwards \& Hame. Le type de cette dernière espèce proviendrait du «cap Natal» qu'il est difficile de localiser. Tmel (1928) croyait cette localité située en Afrique du Sud (où Gardiner 1904, p. 123, avait déjà mentionné un cap de ce nom), et Monod (1954) au Brésil. Or, selon LABOREL (1973) la localité type serait plutôt à chercher dans le golfe de Guinée. O. fissipara n'est pas le seul représentant 
de ce genre dans l'Atlantique tropical africain: Thiel (1928) y a décrit une espèce distincte, O. africana, en provenance du Congo/Brazzaville.

LABOREL (1973) a étudié récemment la variabilité de ces deux espèces africaines et a établi leur synonymie. Il propose d'ailleurs d'élever le sous-genre Schizoculina WeLls 1937, au rang d'un grenre indépendant pour séparer les deux espèces ouestafricaines des espèces américaines au niveau générique.

Oculina virgosa Squtres 1958 (voir Ralph \& Squtres 1962, Squires \& Keyes 1967), de l'extrême nord de la Nouvelle Zélande, paraît très différente des Oculina atlantiques (américaines et africaines). L'appartenance générique de cette forme reste à déterminer.

Des formes fossiles du genre Oculina ont été signalées en Europe, mais surtout en Amérique du Nord et en Amérique du Sud (parmi celles-ci O. patagonica ANGelis, maintenant retrouvée vivante en Méditerranée). Plusieurs espèces fossiles du genre Oculina auraient été trouvées également dans la région australe-asiatique (SQuIRES 1958, RALPH \& SQUTREs 1962).

Dans la littérature on trouve des indications contradictoires sur la nature, hermatypique ou ahermatypique, des espèces du genre Oculina. Selon Vaughan \& Wells (1943) et WELLs (1956) le genre Oculina comprend des espèces hermatypiques et ahermatypiques, tandis que CHEvalien (1961, p. 209) prétendait que «les Oculinidae ne possèdent pas de zooxanthelles symbiotiques et ne renfermaient pas de formes hermatypiques». Or, DUERDEN (1902) a rapporté l'abondance des zooxanthelles chez O. diffusa LAMARck, espèce vivant à faible profondeur aux Antilles. Pour l'espèce O. arbuscula Agassiz (North Carolina, South Carolina), les indications sont contradictoires: McCloskey (1970) l'a présentée comme hermatypique, MacintYre \& PILKEY (1969) comme ahermatypique. Les deux espèces ouest-africaines (genre Schizoculina, selon LABOREL 1973) possèdent bien des zooxantheiles. Enfin, la récolte, à Savona, de O. patagonica ANGelis (première récolte de matériel vivant) permet de savoir que cette espèce est hermatypique.

\section{Oculina patagonica - espèce de l'Amérique du Sud}

O. patagonica fut décrite et figurée pour la première fois, de façon bien reconnaissable, par ANGELIS (1908) qui considérait alors cette forme comme une "variété» nouvelle (var. patagonica) de $O$. mississipiensis CONRAD, espèce fossile de l'Amérique du Nord. Ce rapprochement est pourtant abusif car les deux formes sont très différentes si on se référe, pour $O$. mississipiensis, à la description détaillée et bien illustrée que Vaughan (1900) en a donnée. La description de la nouvelle espèce sud-américaine (comme var. patagonica) par ANGelis était basée sur des échantillons fossiles des provenances suivantes: (a) Sud de Colhue Huapi, Patagonien inférieur (coll. C. Ameghino); (b) Puerto Militar, Bahia Blanca, Pampanéen; (c) Isla Chica (coll. TOURNOUËR).

SQuires (1963) a redécrit la même espèce sous le nom de Astrangia patagonica n. sp. Il en admettait, inexplicitement, l'identité avec $O$. mississipiensis var. patagonica ANGelis, dont il n'avait d'ailleurs pas revu le matériel. Squrres a basé la nouvelle 
description sur des échantillons des provenances suivantes: (a) Monte Hermoso, Pleistocène? Holocène? (coll. C. AmeghINo); (b) matériel étiqueté (provenance?): Caride - Angel Zotta; (c) Playa del Barco, Dorrego (Buenos Aires), Postpampanéen, Holocène (coll. M. Doello-Jurado 1924); (d) Puerto Belgrano, Bahia Blanca.

La plupart des échantillons mentionnés par ANeelis (1908) et par Squires (1963) se trouvent au musée de Buenos Aires. J'ai pu examiner un de ces échantillons (celui figuré par Squires 1963, Fig. 10-11; Mus. Argent. Ci. nat., no 9867), ici à nouveau figuré (Fig. 2e). En principe, le Muséum National d'Histoire Naturelle, à Paris, devrait également posséder des échantillons fossiles provenant de l'Argentine (Isla Chica, coll. Tournouër, échantillons mentionnés par ANGelis 1908). Toutefois, étant donné l'état général d'abandon et de dégradation dans lequel se trouvent, depuis longtemps, les collections du Muséum, il a été impossible de retrouver ces échantillons.

Les échantillons de O. patagonica étudiés par ANGelis (1908) et par SQuires (1963) proviennent de diverses localités situées entre le sud de la province de Buenos Aires et celle de Comodoro Rivadavia (en Patagonie), grossièrement entre $39^{\circ} \mathrm{S}$ et $46^{\circ} \mathrm{S}$. Tous ces échantillons étaient plus ou moins roulés, subfossiles ou fossiles. Ils datent, pour les plus anciens, du Pleistocène. Aucun échantillon n'avait été récolté en milieu marin, mais certains ont toutefois été trouvés non loin de la mer et pourraient avoir été rejetés à une époque subactuelle. SQutres (1963) a très justement posé la question de savoir si l'espèce était encore vivante sur les côtes argentines.

Cependant, il se peut que $O$. patagonica ne vive plus aux latitudes où lespèce avait été trouvée à l'état subfossile ou fossile. La récolte de Astrangia ratbbuni VAUGHan 1906, à l'état vivant à Mar del Plata, et subfossile à la Terre de Feu (Squires 1963) pourrait indiquer que certaines espèces (dont $O$. patagonica) avaient pu progresser vers le sud lors d'une période de réchauffement général, peut-être assez récent, dans ces latitudes.

En attribuant l'espèce O. patagonica ANGelss au genre Astrangia, SQumes (1963) n'a pas cherché à connaître ses affinités dans le genre Oculina, mais il a insisté sur les différences entre "Astrangia» patagonica et deux espèces authentiques du genre Astrangia déjà connues sur les côtes atlantiques de l'Amérique du Sud (A. braziliensis VAUGHAN et $A$. ratbbuni VAUGHAN) et comparé leurs aires géographiques respectives.

Pax (1910, p. 74) et Gravier (1914, p. 121), en énumérant les espèces de Madréporaires connues dans les régions antarctiques et subantarctiques, ont mentionné Astrangia sp. pour le détroit de Magellan ou la Terre de Feu (sans le moindre détail). Squires (1961, p. 20) remarquait à ce sujet: «an occurence of Astrangia mentioned by PAX (1910) and Gravier (1914) from Magellan Strait would be of considerable interest if it could be verified, but both are citations of a previous record unknown to me». Lors de la discussion sur les affinités et la distribution de son «Astrangia» patagonica, SQUIRES (1963) revenait à nouveau sur cette signalisation imprécise. Or, il semble bien que, sans en citer l'auteur, PAX et Gravien avaient simplement repris une indication donnée par VERRILl: ce dernier (VERRILL 1869, p. 526) avait mentionné une espèce non identifiée du genre Astrangia pour le détroit de Magellan (sans aucun autre détail). Or, auparavant ce même auteur avait déjà publié une liste de coraux (et d'autres invertébrés) du Museum of Comparative Zoology, Cambridge/Mass. (Verrill 1864); il était donc familier avec la collection de ce musée. Ce même musée 
posséde en effet de nombreux échantillons subfossiles de Astrangia ratbbuni Vaughan, récoltés à la Terre de Feu par la célèbre U.S. Exploring Expedition 1836-1842 (récolte mentionnée par SQUIREs 1963). Ainsi VERRILl avait probablement fait allusion à ces mêmes échantillons ultérieurement identifiés par SQurRes.

Contrairement à ce que pourraient faire penser les réflexions de SQuires et le nom bien suggestif de Oculina (ou Astrangia) patagonica, on n'a donc aucune indication selon laquelle l'espèce en question aurait été trouvée au détroit de Magellan ou à la Terre de Feu.

\author{
Oculina patagonica- \\ redescription d'après le matériel méditerranéen
}

La découverte d'une colonie vivante de Oculina patagonica en Méditerranée permet de redécrire l'espèce avec plus de détail, et d'après du matériel plus représentatif que les échantillons argentins, fossiles ou subfossiles, et plus ou moins abimés. En particulier, il est maintenant possible de caractériser les parties molles, et ceci pour la première fois.

Les tissus vivants de O. patagonica sont bourrés de symbiontes du groupe des Dinozooxanthelles et proches de Symbiodinium microadriaticum (FrevdenTHAL). Ces organismes mis en cultures sont étudiés par G. Duclaux, du Laboratoire de Biologie Végétale Marine (Université de Paris). Lorsque des fragments de la colonie sont conservés dans l'alcool, les pigments des zooxanthelles donnent au liquide une très forte coloration brun-verdâtre.

A l'exception de la bouche des polypes et de leurs tentacules, plus clairs, la coloration de la colonie est normalement d'un brunâtre difficile à définir, pouvant aller vers le mauve. A l'abri de la lumière, lorsque le Scléractiniaire a envahi une anfractuosité du substrat ou l'intérieur d'un grand Balane mort, les tissus sont clairs, plutôt roses. Cette dernière coloration semble être due à un pigment propre au Scléractiniaire tandis que la coloration brune générale des parties exposées à la lumière (surtout du coenosarc) est essentiellement due aux zooxanthelles. La coloration de la bouche des polype, en forme de fente transversale, peut aller vers le jaune ou l'orangé. Les tentacules sont plutôt d'un bleu-grisâtre dont l'intensité dépend de l'état de contraction. Les tentacules sont marqués de nombreux petits points blancs qui semblent correspondre à des concentrations de nématocystes, tout comme, par ailleurs, le bout terminal, également blanc et légèrement épaissi, de ces tentacules (Fig. 1a-c).

Normalement, il y a 24 tentacules disposés assez clairement en deux cercles autour du péristome des polypes (Fig. 1a, b). Même en pleine extension, ces tentacules sont relativement courts et trapus, en comparaison de ceux de la plupart des especes méditerranéennes de Scléractiniaires. Lorsque les polypes sont plus ou moins rétractés dans la cavité calicinal du squelette et l'anneau concave qui l'entoure normalement à la surface du coenosteum (voir plus loin), les extrémités terminales, blanches et légèrement épaissies, des tentacules se trouvent en deux cercles concentriques distincts au niveau de l'orifice du calice (Fig. 1 b). 


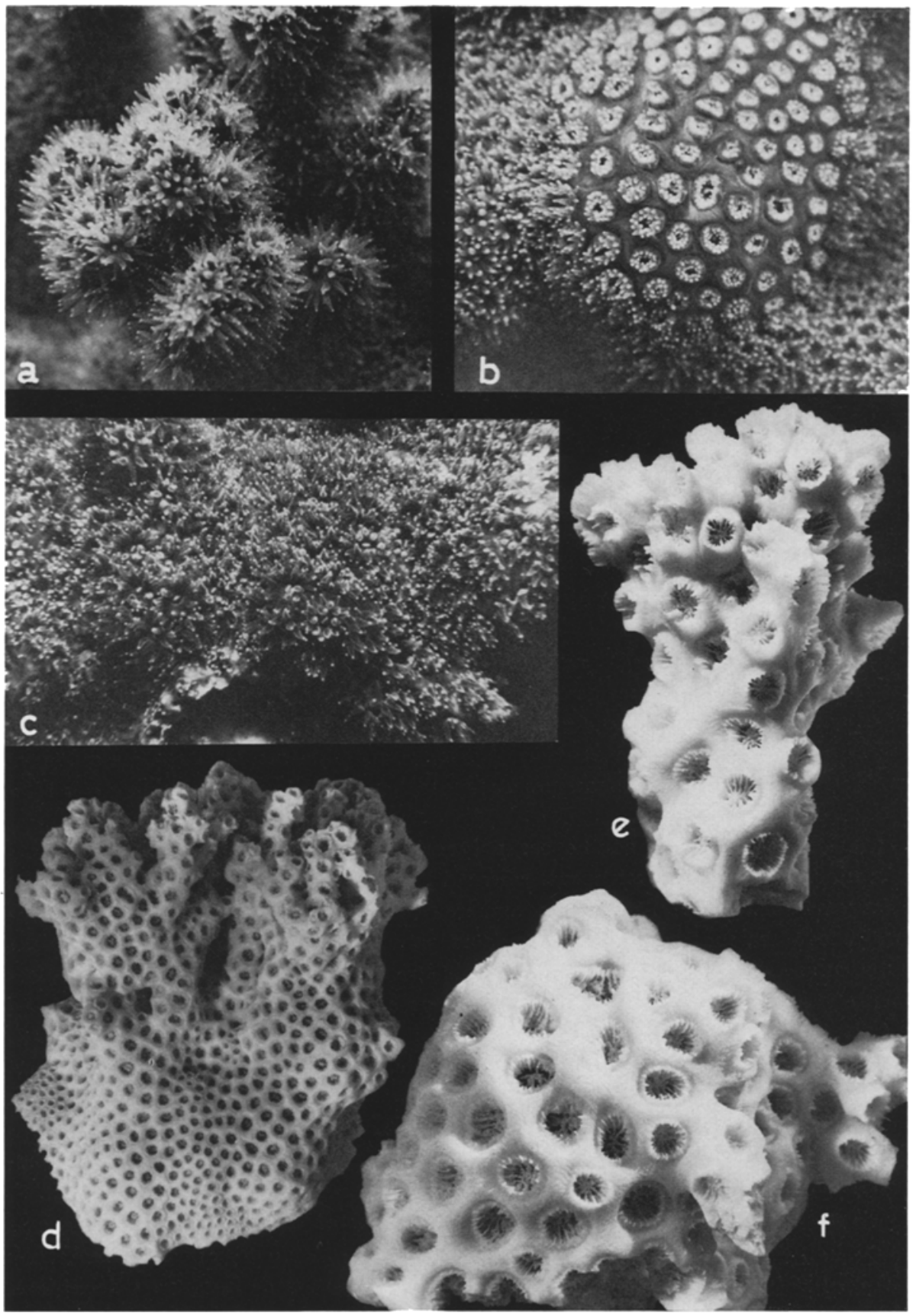

Fig. 1: Oculina patagonica de la Méditerranée. a Détail de la colonie (Savona, 23. X. 1972) zone à petites branches dressées, polypes épanouis (1,7:1). $b$ Détail de la colonie (Savona, 23. X. 1972) zone plate à bosselé, polypes retractes $(1,7: 1)$. $c$ Fragment de la colonie transplanté à Marseille (île de Riou, 5. XI. 1972) (1,5:1). d Grand fragment à petites branches dressées $(0,7: 1)$. e Branche isolée (2:1). $f$ Fragment arrondi, épais; à droite le début de deux branches $(2: 1)$ 


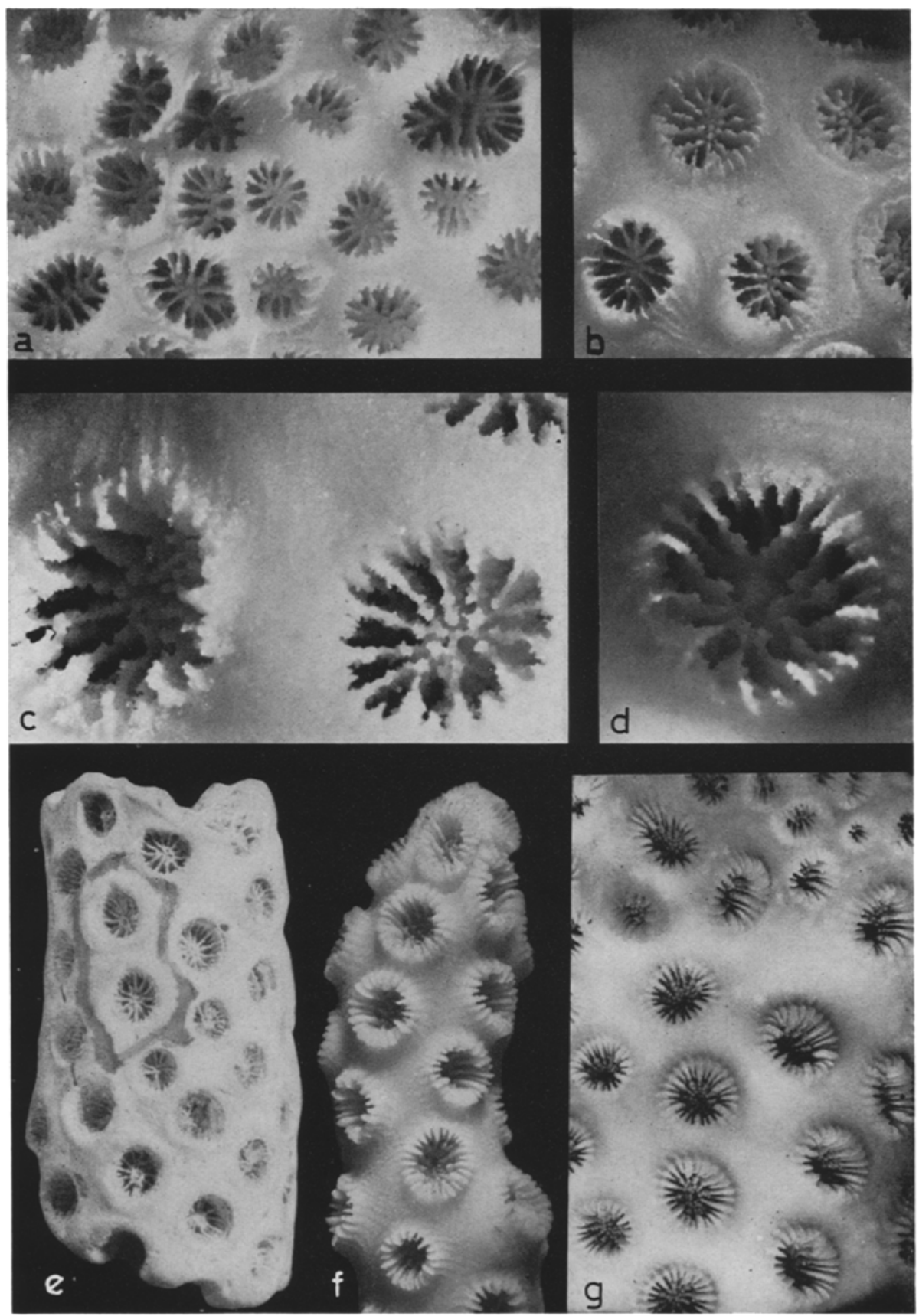

Fig. $2 a-d$ : Oculina patagonica de la Méditerranée. Diverses vues détaillées montrant la variabilité des calices $(a$ 5,2:1, $b 6,2: 1$, c 9,5:1, $d$ 9,5:1). e Oculina patagonica de l'Argentine (=Astrangia patagonica SQuires 1963; paratype) Mus. Argent. Ci. nat. no 9867 (2:1). f Oculina arbuscula de North Carolina (échantillon reçu de W. K. KIRBY-SMITH), branche terminale $(3,6: 1)$; g partie basale encroûtante de la colonie $(3,6: 1)$ 
Suivant les secteurs, la colonie est encroûtante, bosselée ou buissonnante, mais non arborescente. Le squelette est mince dans les zones périphériques, récentes, mais très épaissi dans les zones plus anciennes où l'épaisseur peut atteindre plusieurs centimètres (Fig. 1d-f). Les branches sont courtes et épaisses, peu et irrégulièrement ramifiées (Fig. 1d, e), et ne représentent pas l'essentiel de la colonie, mais simplement des excroissances. Dans les zones très épaissies, les mêmes calices se poursuivent sur plusieurs centimètres de hauteur (visibles par section longitudinale), mais des planchers transversaux (dissépiments) subdivisent les loges interseptales à des niveaux successifs.

$\mathrm{Si}$, dans l'ensemble, l'aspect de la colonie est peu uniforme, la disposition des corallites, les uns par rapport aux autres, l'est également. Les parties épaissies de la colonie, plutôt planes ou bosselées, présentent certainement le mieux les caractères particuliers de l'espèce: les corallites s'y trouvent entièrement immergés dans le coenosteum massif (au point que le bord supérieur de leurs septes dépasse à peine le niveau du coenosteum) et chaque calice est ici entouré d'une dépression étroite en anneau (Fig. 1d, f). Cet anneau lisse est très net, tout en étant peu profond (plus rarement, il forme un véritable sillon). Les calices sont ronds, allongés ou vaguement polygonaux, tantôt serrés, tantôt plus espacés. La distance qui les sépare peut varier de moins de la moitié de leur diamètre jusqu'à plus d'une fois leur diamètre (Fig. 1f, 2a). En général, le diamètre des calices varie autour de $2,5 \mathrm{~mm}$, mais il y en a qui sont beaucoup plus petits (de l'ordre de $1 \mathrm{~mm}$ ). Lorsque les calices sont très serrés, le coenosteum qui les sépare peut constituer une sorte de crête, puisqu'il est plus élevé que leur périphérie en anneau. Lorsque deux parties de la colonie, venant de directions différentes, se rencontrent en fusionnant, il peut y avoir une limite très nette entre une zone à calices petits et une zone à calices plus grands. En général, le coenosteum est à peu près lisse, à part une très fine granulation. Il n'y a pas de véritables côtes; toutefois, la surface peut être légèrement plissée, marquée par des stries dans un sens préférentiel.

Dans les zones périphériques où la colonie s'accroît en largeur, par exemple en s'étalant sur des Balanes, le bord distal des corallites est sensiblement élevé au dessus du coenosteum encore très mince. De même, à l'extrémité des branches, les corallites peuvent être libres, sur une hauteur de 1 à $2 \mathrm{~mm}$ (Fig. 1e). Dans ces deux dernières zones, les calices ne sont alors pas entourés de l'anneau légèrement concave (et si caractéristique des zones épaissies, planes ou bosselées) mais peuvent comporter de véritables côtes. C'est dans la partie distale des branches que le bourgeonnement extracalicinal est évident, tandis que le bourgeonnement intracalicinal peut être observé, parfois, dans les zones de la colonie plus épaissies, plates ou bosselées. Il y a donc une différence considérable entre les extrémités terminales des branches et les zones encroûtantes épaissies (Fig. 1e, f).

En général, les calices comportent 24 septes étroits, dont 12 septes principaux fusionnés au centre, et 12 septes libres, alternant régulièrement. La partie supérieure des septes principaux est verticale, la partie inférieure s'incline vers l'axe et comporte plusieurs lobes, en général dirigés vers le centre. Il n'y a pas de columelle bien individualisée. Les papilles de la columelle sont des lobes du même type que ceux du bord des septes principaux, mais à un niveau inférieur (plus ancien) et déjà intégrés dans la structure centrale. Les septes sont minces, espacés, à granulation peu développée 
sur leurs faces latérales. Les lobes libres des septes et la columelle varient considérablement d'un calice à l'autre, comme le montrent les illustrations (Fig. 2a-d).

Du matériel représentatif prélevé à Savona a été déposé au British Museum. (Nat. Hist.), London (1973.5.21.2).

\section{Affinités de Oculina patagonica}

C'est au niveau des extrémités de ses branches irrégulières, par ses corallites en partie dégagés et dépassant le coenosteum, que $O$. patagonica ressemble le plus aux nombreuses espèces du genre Oculina décrites dans les mers américaines et aux deux espèces ouest-africaines (genre Schizoculina, selon LABorel 1973). Par contre, ses calices entièrement immergés dans le coenosteum des parties basales épaissies, calices entourés d'un anneau lisse et légèrement enfoncé, constituent un caractère très particulier et distinctif.

Ignorant les descriptions par ANGelis (1908) et SQuires (1963), Rossi (1969, p. 151) a assimilé O. patagonica (colonie découverte à Savona par Luigr Morra) à "Oculina arbuscula» du golfe de Guinée (en réalité O. fissipara Milne Edwards \& HaIme). Or, ce rapprochement doit surprendre car, pour les raisons évoquées, le Scléractiniaire de Savona se distingue nettement de l'espèce ouest-africaine, ainsi que du vrai $O$. arbuscula Agassiz. Cette dernière espèce, connue dans une zone assez étroite, entre la cap Hatteras (North Carolina) et Charleston (South Carolina) (cf. McCloskey 1970) se rapproche beaucoup des autres espèces américaines. A titre de comparaison, je présente ici deux vues détaillées de $\mathrm{O}$. arbuscula, en provenance de North Carolina (branche distale: Fig. 2f; partie basale: Fig. 2g).

WeLLs (1937) a proposé de regrouper, dans le sous-genre Schizoculina, les espèces africaines caractérisées par la plus grande fréquence du bourgeonnement intracalicinal. Ce caractère, à lui seul, paraît assez vague et loin d'être déterminant: j'ai ainsi constaté l'existence du bourgeonnement intracalicinal dans diverses espèces méditerranéennes appartenant à des genres où ce type de multiplication n'avait jamais été signalé. LABOREL (1973) a repris la proposition de WeLls, mais en apportant des arguments supplémentaires, il a fait de Scbizoculina un genre indépendant. Pour LABOREL, le genre Schizoculina comprendrait des Oculinidae plutôt archaïques et assez proches des Astrangiidae, caractérisés par une grande variabilité de la colonie en fonction du milieu ambiant et par une plus grande fréquence du bourgeonnement intracalicinal.

En ce qui concerne Oculina patagonica, il parâ̂t significatif que cette espèce ait été attribuée, indépendemment, aux genres Oculina (par Angelrs 1908) et Astrangia (par Squires 1963). Cependant, en attendant une étude plus détaillée des formes tropicales à redistribuer entre Oculina et Scbizoculina, il est préférable de retenir ce premier nom générique pour l'espèce introduite en Méditerranée. 


\section{RÉSUMÉ}

1. En 1966, une colonie de l'espèce hermatypique Oculina patagonica a été découverte vivant sur un rocher (profondeur $0,5-2 \mathrm{~m}$ ) à environ $1 \mathrm{~km} \mathrm{du}$ port de Savona (golfe de Gênes, Italie). Elle a été étudiée à nouveau en 1971 et 1972.

2. La température de l'eau de mer dans le secteur varie entre environ $11^{\circ} \mathrm{C}$ et $26^{\circ} \mathrm{C}$. Pendant la plus grande partie de l'année elle reste bien inférieure à la température considérée généralement comme nécessaire à la croissance et même à la survie de Scléractiniaires hermatypiques.

3. Malgré la pollution locale favorisant des populations denses et prospères de Balanes et d'Ascidies, la colonie (dimensions: $1,2 \mathrm{~m} \times 1,5 \mathrm{~m}$ ) prospère et tend a recouvrir ces autres organismes.

4. Le peuplement benthique sur roche entourant la colonie comprend diverses espèces animales et algales introduites, dont certaines déjà largement répandues dans les peuplements portuaires de la Méditerranée. Cependant, la plus importante de ces espèces introduites, l'Ascidie Microcosmus exasperatus, n'avait pas encore été signalée en Méditerranée.

5. Des échantillons du Scléractiniaire prélevés à Savona ont été installés à Marseille où ils ont grandi pendant les 15 mois de l'expérience, notamment dans l'eau pure du large.

6. Auparavant, $O$. patagonica n'était connue que d'après des fragments fossiles ou subfossiles trouvés en Argentine, toujours en milieu terrestre.

7. Il ist probable que l'espèce vit, à l'époque actuelle, sur les côtes atlantiques de l'Amérique du Sud d'où elle aurait été introduite en Méditerranée sur une coque de bateau.

Remerciments. Je n'aurais pas pu commencer la présente étude si G. SCHEER ne m'avait pas mis en contact avec L. Morra, et si ce dernier ne m'avait pas communiqué ses observations et emmené plonger avec lui à Savona. Divers spécialistes m'ont prété leur concours pour l'identification des espèces récoltées: F. Arnaud (Pycnogonides), P. M. Arnaud (Mollusques), G. Bellan (Polychètes autres que Serpulidae), D. Bellan-Santint (Amphipodes), C. F, Boudouresque (Algues), G. Cherbonnier (Echinodermes), J. Forest (Décapodes), J. G. Harmelin (Bryozoaires), M. A. Mrzler (Tanaidacés et Isopodes), C. Monniot (Ascidies), J. PiCard (Hydraires), G. Relini (Cirripèdes). G. Relini a également fourni des données hydrologiques. J. G. Harmelin a pris les photos sous-marines et aidé à la transplantation des échantillons de Savona à Marseille. W. K. Kirby-Smith et J. W. Wells m'ont procuré des échantillons d'espèces américaines du genre Oculina, et un échantillon de Oculina patagonica m'a été prêté par E. Martinez Fontes (Museo Argentino de Ciencias Naturales). Enfin, un séjour au British Museum (Nat. Hist.), financé par le British Council, et l'hospitalité de la Coelenterate Section ont largement facilité le présent travail.

\section{LITTERATURE CITEEE}

ABE, N., 1937. Post-larval development of the coral Fungia actiniformis var. palawensis Doederlein. Palao trop. biol. Stn Stud. 1 (1), 73-93.

- 1939. Ecological studies on Rbizopsammia minuta var. mutsuensis YABE \& EguchI In: Jubilee Publ. Prof. H. YABE 60th Birthday, 1, 175-187 [en japonais, avec résumé anglais]. 
Agassiz, L., 1880. Report on the Florida reefs, accompanied by illustrations of Florida reef corals with an explanation of plates by E. F. PourTalès. Mem. Mus. comp. Zool. Harv. $7(1), 1-61$.

Angelis d'Ossat, G. DE, 1908. Altri zoantari del terziario della Patagonia. An. Mus. nac. Buenos Aires, (Ser. 3) 9, 93-102.

Chevalier, J. P., 1961. Recherches sur les Madréporaires et les formations récifales du miocène de la Méditerranée. Mém. Soc. géol. Fr. (N. S.) 40 (93), 1-562.

Duchassaing, P. \& Mrchelotti, J., 1864. Supplément au mémoire sur les Coralliaires des Antilles. Memorie Accad. Sci. Torino 23, 97-206.

Duclaux, G. \& Lafargue, F., 1973. Madréporaires de Méditerranée occidentale. Recherche des zooxanthelles. Compléments morphologiques et écologiques. Vie Milieu (A) 23, 45-63.

Duerden, J. E., 1902. West Indian madreporarian polyps. Mem. natn Acad. Sci. 8 (7), 399-597.

Duncan, P. M., 1876. Notices of some deep-sea and littoral corals from the Atlantic Ocean, Caribbean, Indian, New Zealand, Persian Gulf, and Japanese Seas. Proc. zool. Soc. London 1876, 428-442.

EhrenBerg, C. G., 1834. Beiträge zur physiologischen Kenntnis der Corallenthiere im allgemeinen, und besonders des rothen Meeres, nebst einem Versuche zur physiologischen Systematik derselben. Abh. preuß. Akad. Wiss. (Phys.-math.) 1832, 225-380.

Gardiner, J. S., 1904. South African corals of the genus Flabellum, with an account of their anatomy and development. Mar. Invest. S. Afr. 2 (6), 117-154.

Gravier, C., 1909. Madréporaires des îles San Thomé et du Prince (Golfe de Guinée). Annls Inst. océanogr., Monaco 1 (2), 1-28.

- 1914. Madréporaires. Deuxième Exp. Antarct. Franç., 1908-1910. Paris, 119-133.

LABOREL, J., 1961. Sur un cas particulier de concrétionnement animal. Concrétionnement à Cladocora cespitosa L. dans le Golfe de Talente. Rapp. P.-v. Réun. Commn int. Explor. scient. Mer méditerr. 16 (2), 429-432.

- 1973. West African corals. An hypothesis on their origin. Second International Symposium on Coral Reefs, Australia 1973 (sous presse).

LAMARCK, J. B. P. A. DE, 1810. Histoire naturelle des animaux sans vertèbres. Paris, 2, 1-568.

Le Cointre, G., 1950. Coquilles remarquables du quaternaire marocain. J. Conch., Paris 90, 240-244.

- 1952. Recherches sur le Néogène et le Quaternaire marins de la côte atlantique du Maroc. T. 2. Paléontologie. Notes Mém. Serv. géol. Maroc. 99, 1-170.

Lesueur, C. A., 1820. Description de plusieurs animaux appartenant aux polypiers lamellifères de M. le Chev. de Lamarck. Mém. Mus. natn. Hist. nat., Paris 6, 271-298.

LiNNE, C., 1758. Systema Naturae. Stockholm 1, 1-823.

McCloskey, L. R., 1970. The dynamics of the community associated with a marine Scleractinian coral. Int. Revue ges. Hydrobiol. 55, 13-81.

MacINTYre, I. G., 1970. New data on the occurence of tropical reef corals on the North Carolina continental shelf. J. Elisha Mitchell scient. Soc. 86, 178.

- \& Pilkey, O. H., 1969. Tropical reef corals: tolerance of low temperatures on the North Carolina continental shelf. Science, N. Y. 166, 374-375.

Mrlne Edwarus, H. \& Hame, J, 1850. Recherches sur les polypiers. Cinquième mémoire. Monographie des Oculinides. Ann. Sci. nat., Paris (Zool.) 13, 63-110.

- - 1850-1854. A monograph of the British fossil corals. London, $322 \mathrm{pp}$.

- - 1857. Histoire naturelle des Coralliaires. Classification et description des zoanthaires sclérodermés de la section des Madréporaires apores. Paris, 1-633.

Monod, T., 1954. Sur deux Madréporaires ouest-africains. Annls Mus. r. Afr. cent. (Zool.) 1 , 222-230.

PAX, F., 1910. Steinkorallen. Dt. Südpol.-Exped. 12 (1), 63-76.

Porzat, C, 1970. Hydrodynamisme et sédimentation dans le Golfe de Gabès (Tunisie). Téthys 2, 267-295.

- 1972. Méthodes d'évage des Gastéropodes Opisthobranches de petites et moyennes dimensions. Mise au point d'un circuit fermé en eau de mer. Premiers résultats. T'éthys 4, 251-267. 
Pourtalès, L. F. DE, 1869. Contributions to the fauna of the Gulf Stream at great depths (Ser. 2). Bull. Mus. comp. Zool. Harv. 1, 121-142.

- 1871. Deep Sea corals. Mem. Mus. comp. Zool. Harv. 2 (4), 1-93.

QueLCH, J. J., 1886. Report on the reef corals collected by H. M. S. Challenger during the years 1873-76. Rep. scient. Results Voyage H. M. S. Challenger (Zool.) 16 (3), 1-203.

Ralph, P. M. \& SQuires, D. F., 1962. The extant Scleractinian corals of New Zealand. Zool. Publ. Victoria Univ., Wellington 29, 1-19.

Ranson, G., 1943. Les types de Madréporaires (Hexacoralliaires) actuels du Muséum d'Histoire Naturelle (chaire de Malacologie). I. Types des espèces décrites pour la première fois par Lamarck. Bull. Mus. natn. Hist. nat., Paris 15, 115-122.

Reurni, G., 1968. Segnalazione di due Cirripedi nuovi per l'Adriatico. Boll. Soc. adriat. Sci. nat. 56, 218-225.

Rossr, L., 1969. Considerazioni zoogeografiche sul bacino N. W. del Mediterraneo con particolare riguardo al Mar Ligure. Archo bot. biogeogr. ital. 45 (4), 139-152.

Squires, D. F., 1958. The Cretaceous and Tertiary corals of New Zealand. Bull. geol. Surv. N. Z. 29, 1-107.

- 1961. Deep-sea corals collected by the Lamont Geological Observatory. 2. Scotia Sea corals. Am. Mus. Novit. 2046, 1-48.

- 1963. Madreporas Rizangidas fosiles y vivientes de la Argentina. Neotropica 9 (19), 9-16.

- \& Keyes, I. W., 1967. The marine fauna of New Zealand: Scleractinian corals. Bull. N. Z. Dep. scient, industr. Res. 185, 1-46.

ThıеL, M. E., 1928. Madreporaria. In: Beiträge zur Kenntnis der Meeresfauna Westafrikas. Hrsg. von W. Mrchaelsen. Friederichsen, Hamburg 3, 251-350.

- 1941. Madreporaria. Mém. Mus. r. Hist. nat. Belg. (Sér. 2) 21, 1-28.

VAUGhan, T. W., 1900. The Eocene and Lower Oligocene coral faunas of the United States with descriptions of a few doubtfully cretaceous species. Monogr. U. S. geol. Surv. 39, $1-263$.

- 1901. The stony corals of the Porto Rican waters. Bull. U. S. Fish Commn 1900, 289-320.

- \& WeLLS, J. W., 1943. Revision of the suborders, families and genera of the Scleractinia. Spec. Pap. geol. Soc. Am. 44, 1-363.

Verrill, A. E., 1864. List of polyps and corals sent by the Museum of Comparative Zoology to other institutions in exchange, with annotations. Bull. Mus. comp. Zool. Harv. 1, 29-60.

- 1868-1870. Review of the corals and polyps of the West coast of America. Trans. Conn. Acad. Arts Sci. 1, 377-558.

- 1901. Comparisons of the Bermudian, West Indian, and Brazilian coral faunas. Trans. Conn. Acad. Arts Sci. 11, 170-206.

WelLs, J. W., 1937. New genera of recent and fossil corals. J. Paleont. 11, 73-77.

- 1956. Scleractinia. In: Treatise on invertebrate paleontology. Ed. by R. C. Moore. Geol. Soc. Am., New York F, 328-444.

- 1957. Corals (annotated bibliography of ecology). In: Treatise on marine ecology and paleoecology. Ed. by J. W. Hedgreth. Geol. Soc. Am., Washington 1, 1087-1104.

Zrrrowius, H., 1973. Remarques sur trois espèces de Serpulidae acclimatées en Méditerranée: Hydroides dianthus (VerRILL 1873), Hydroides dirampha Mörch 1863, et Hydroides elegans (Haswell 1883). Rapp. P.-v. Réun. Commn int. Explor. scient. Mer Méditerr. 21, 683-686.

Adresse de l'auteur: H. Zibrowrus

Station Marine d'Endoume

Rue Batterie des Lions

F-13007 Marseille

France 